\title{
"Yo acojo, tú agrupas, ella compensa": Análisis comparado de la política de integración del alumnado inmigrante en tres Comunidades Autónomas
}

\section{'I receive, you gather, she compensates': Comparative analysis of the politics of integration of immigrant school children in three Autonomous Regions}

\author{
María Jesús MARTÍNEZ-USARRALDE, Carmen María FERNÁNDEZ-GARCÍA y \\ Amalia AYALA-DE LA PEÑA \\ Universitat de València, Universidad de Oviedo y Universidad de Murcia
}

Recibido: Diciembre 2014

Evaluado: Abril 2015

Aceptado: Junio 2015

\section{Resumen}

Con la Ley Orgánica de Mejora de la Calidad Educativa vuelve a emerger uno de los temas educativos más oportunos: la toma de decisiones en torno a las políticas educativas de atención al alumnado inmigrante. El momento supone una excelente excusa para, desde el prisma que nos ofrece la Educación Comparada, revisar cómo el colectivo de infancia inmigrante ha sido educado desde tres modelos de gestión educativa representados por tres Comunidades Autónomas: Comunidad Valenciana, Cataluña y Andalucía. El artículo se sitúa en el análisis de las medidas que se han articulado en los tres casos como respuesta a su recepción, acogida y escolarización, utilizando una metodología cualitativa basada en el método comparado: en el estudio descriptivo (fase analítica), se realiza una exploración explicativa de las medidas articuladas en torno a las políticas de gestión del alumnado inmigrante, mediante el análisis de contenido de fuentes primarias y secundarias. Con respecto a los resultados, en la fase sintética, tras haber yuxtapuesto los principales criterios que asemejan y también dirimen a los tres modelos, se reconoce cómo las tres Comunidades Autónomas presentan particularidades, fruto de trayectos históricos propios, coyunturas económicas y políticas e intenciones educativas y sociales, influidas por contextos en los que algunos factores han tenido un peso específico importante. Además del protagonismo institucional, se detectan diferencias notables en las medidas concretas adoptadas, pero también coincidencias, como las políticas que apoyan económicamente la lengua de la comunidad autónoma. Finalmente, en la fase de conclusiones comparadas, se plantean una serie de elementos de los que se deberían dotar las Comunidades Autónomas y que se hallan presentes en algunos modelos analizados. La atención a la lengua 
que porta el alumnado o el cuidado por incluir una educación intercultural en los planes de los centros educativos son dos de las acciones más significativas.

Palabras clave: alumnado inmigrante, integración educativa, política educativa, educación formal, educación comparada.

\begin{abstract}
With the passing of the la Ley Orgánica de Mejora de la Calidad Educativa [Reform Act of Improvement of Quality in Education] reemerges one of the timeliest educational issues: decision-making in the field of educational policy that takes care of immigrant students. The present moment is an excellent excuse for, from the perspective that Comparative Education offers, the revision of how the collective of immigrant children has been educated through three models of educational management represented by three Autonomous Communities: Valencia, Catalonia and Andalusia. The article focuses on the analysis of the measures taken in these three cases concerning the reception, the welcoming, and the schooling of immigrant children, using a qualitative methodology based on the comparative method: the descriptive study (analytical phase) presents an explanatory scanning of the measures adopted on management policies of immigrant students, through the content analysis of primary and secondary sources. Regarding results, in the synthetic phase, after juxtaposing the principal criteria which are similar or different in the three presented models, it must be admitted that the three Autonomous Communities have their own singularities, which derive from their own historical backgrounds, economic and political conjunctures, and their educational and social aims, influenced by contexts where certain factors have had a significant weight. Apart from institutional prominence, remarkable differences appear in certain measures, but also coincidences, as for example policies that support the language of the region in each of the presented cases. Finally, the section of comparative conclusions proposes a number of elements which the autonomous communities should acquire, which are present in some of the analysed models. Attention to the language of immigrant students and care for the inclusion of an intercultural education in the curricula of the education centres, are two of the most significant actions.
\end{abstract}

Keywords: immigrant students, educational integration, educational policy, formal education, comparative education.

La Ley Orgánica de Mejora de la Calidad Educativa establece en su Preámbulo la necesidad de superar las rigideces de un sistema educativo que puede generar la exclusión de aquellos alumnos cuyas expectativas no se adecúan al marco establecido. En este perfil, aunque no exclusivamente, es posible reconocer a algunos de los alumnos inmigrantes que llegan a nuestro sistema educativo en momentos variables del año y con un dominio muy heterogéneo del idioma vehicular en el que se imparten las clases. Dado el carácter descentralizado del sistema educativo español y la cesión de competencias a las respectivas Comunidades Autónomas, resulta sugerente analizar cómo afrontan éstas la atención al alumnado inmigrante y cómo garantizan o apoyan el derecho a la educación de los estudiantes de incorporación tardía o con escaso dominio del idioma en el que se imparten las clases. Más allá de la relevante repercusión educativa que estas medidas poseen, no puede tampoco obviarse la 
importancia de que la institución escolar actúe como elemento mediador en una sociedad que no siempre parece estar preparada para entender y comprender todo lo que rodea al fenómeno migratorio (García, Granados, Olmos y Martínez, 2014).

\section{Semejantes necesidades, diferentes modelos de intervención para la atención a los estudiantes inmigrantes}

La responsabilidad de las Administraciones públicas respecto a la escolarización de este alumnado se materializa en diferentes estrategias por parte de las Comunidades Autónomas que han sido analizadas en la literatura pedagógica reciente (Aja, Arango y Oliver, 2012; Alcalde, 2008; Alegre, Benito y González, 2012; Arroyo, 2012; Del Olmo, 2012; Etxeberría y Elosegui, 2010; Fernández, 2013; García y Carrasco, 2011; García, Rubio y Bouachra, 2008; Grañeras, Vázquez, Parra, Rodríguez, Madrigal y Vale, 2007; Llevot, 2006; Martínez de Lizarrondo, 2009; Martínez Usarralde, 2010; Palaudàrias y Garreta, 2008; Simó, Pamies, Collet-Sabé y Tort, 2014; Siques, Vila y Perera, 2009; Vega, 2005). La multiplicidad en las denominaciones, en las formas de proceder, la temporalidad con que se piensan o el grado de autonomía que se concede a los centros dificulta dibujar un panorama completo de lo que está sucediendo en nuestro entorno más próximo. Esta variabilidad se observa también en el conjunto de los países de la Unión Europea (Comisión de las Comunidades Europeas, 2008; Eurydice, 2009; García y Rubio, 2013; Martínez Usarralde, 2008; Llorent y Terrón, 2013; OECD, 2012) quedando plasmada en las diferentes iniciativas propuestas: acuerdos bilaterales con los países de origen, formación del profesorado o publicación de información sobre el sistema educativo destinada al entorno familiar, entre otros.

En todo caso, se precisa revisar muchos de los planteamientos, pues el apoyo recibido por estos estudiantes no siempre es suficiente ni atiende convenientemente, por ejemplo, a los aprendizajes adquiridos en sus países de origen o a las dificultades para la participación de sus familias en los procesos de enseñanza-aprendizaje (Goenechea, 2005; Huddleston y Niessen, 2011). Esta desventaja educativa de los discentes de origen extranjero fue puesta de manifiesto en el Libro Verde de la Unión Europea sobre Inmigración y Movilidad en el que se destacan no sólo las consecuencias individuales nefastas que implica para estos menores una atención inadecuada sino, además, el lastre para la cohesión, la equidad y la inclusión social (Comisión de las Comunidades Europeas, 2008).

\section{Diseño y metodología}

La elección de la metodología propia de la disciplina científica de la Educación Comparada obedece a que, a través de ella, se despliegan amplias oportunidades de configuración del mundo sociocultural y, en el mismo contexto, demuestran cómo y de qué manera estas realizaciones han seguido la lógica de estructuraciones sistémicas, enriqueciendo así nuestro conocimiento sistemático y subrayando a la vez la idea de apertura evolutiva de la praxis social (Schriewer, 2010).

Con esta aspiración como escenario, podemos remitirnos a uno de los "usos" de la 
Educación Comparada que ya fuera definido por Sadler en 1900, y que no es otro que acercarnos a otras realidades socioeducativas diferentes, a fin de buscar orientaciones alternativas que puedan ser de utilidad y así reinterpretar las problemáticas propias. Dicho con sus propias palabras, en su obra "How far can we learn anything of practical value from the study of foreign systems of education?", texto que sigue erigiéndose en referente valioso, "el valor práctico del estudio, bajo una intención correcta y rigor académico, de los sistemas extranjeros de educación, reside en que servirá para que el nuestro propio sea mejor estudiado y comprendido" (Sadler, 1900: 12). En la actualidad, esta tendencia cobra una especial vigencia, a veces bajo la rúbrica del modelo de "préstamo y copia" y actualmente reconocido bajo el concepto anglosajón de transfer (Halls, 1990; Noah, 1990; Phillips, 2000; Schriewer, 1990), para "tender puentes entre culturas" (Crossley, 2008). Precisamente, como sostiene Larsen (2010) es Cowen quien se centra en reconocer la complejidad intrínseca que conllevan estos estudios, tanto de transferencia como de préstamo (borrowing), y acordamos con ella que más que pensar en que las políticas de transfer son procesos lineales y de una dirección, hoy en día debe considerarse su naturaleza recíproca y circular.

Situándonos en el modelo heurístico del Cubo de Bray \& Thomas (1995) para reflejar los diferentes niveles de un estudio de Educación Comparada, nuestro análisis se insertaría en el nivel 3, denominado de "Provincias o Estados"(diferenciándolo del nivel 2, "países"), conocido también como comparación intranacional (Lê Thànk Kôi, 1981). En efecto, ésta constituye una unidad de análisis propicia cuando, como reconoce Manzón (2007), existen disparidades regionales significativas dentro de un país o dichos países acreditan un alto nivel de descentralización, como ocurre con el caso español. Al mismo tiempo, los niveles de homogeneidad precisos vienen dados por las estructuras de articulación de actuación sociopolítica en el marco de Estado común de las autonomías y por el también común elemento de elevada proporción inmigrante para cada uno de los tres contextos estudiados (Ministerio de Educación y Ciencia, 2014: 8).

\section{Resultados}

\section{Política de integración del alumnado inmigrante en la Comunidad Valenciana}

La Comunidad Valenciana gestiona el ámbito educativo de la inmigración a través de las iniciativas de la Conselleria d'Educació, Cultura i Ciència que se coordina con la Conselleria de Benestar Social. Es, en este sentido, la Orden de 4 de julio de 2001 la que regula la atención al alumnado con necesidades educativas especiales, con la finalidad de compensar las desigualdades, reiterando el principio de igualdad en el ejercicio del derecho a la educación y erigiendo la educación compensatoria como estrategia de acción. Esta ley, que legisla consecuentemente las acciones acometidas con el alumnado inmigrante, establece tres tipos de centros, y, con ello, de políticas educativas, en función del número de estudiantes con necesidades compensatorias que se hallen en los mismos: los Centros de Acción Educativa Singular (CAES) si acogen a más del 30\% de alumnado inmigrante; los Programas de Compensación en centros 
en los que se halle entre el 20 y $30 \%$ del alumnado inmigrante y los centros catalogados como "otros". Más recientemente, se ha podido comprobar cómo en las directrices educativas manejadas desde Conselleria se han creado programas de apoyo a los centros que cuenten con un número significativo de inmigrantes. En dichos programas se trata de impulsar la organización y funcionamiento de dichos centros en tres ámbitos: atención directa al alumnado, intervención con las familias y relación con su entorno; por otro lado, se impulsan medidas de apoyo para mejorar las expectativas escolares y complementar las medidas y recursos de compensación educativa (Carbonell, 2006). Esta iniciativa se complementa con la configuración de las "Aulas PASE", programa temporal de adaptación lingüística para el alumnado extranjero de nueva incorporación al sistema educativo que desconoce la lengua base del programa de educación bilingüe al que queda adscrito (Carbonell, 2006). Esta iniciativa se acompaña además de los "Programas de acompañamiento de educación primaria y educación secundaria", bajo los cuales se ofrece una guía y una orientación personalizada al alumnado con dificultades, después de su horario lectivo.

A partir de la citada Orden, se articula el "Plan inicial para la Atención Educativa para el Alumnado Inmigrante", que se puso en marcha desde el curso académico 2000-2001. Una de sus medidas es la constitución de un Observatorio que proporciona datos actualizados sobre la distribución del alumnado inmigrante de modo que ello facilite la detección de sus necesidades educativas. No menos importancia poseen las Oficinas de Acogida y Atención al alumnado inmigrante y sus familias (OAAI) que actúan como mecanismos de información para la escolarización y colaboran con asociaciones y entidades que trabajan con este colectivo. Como complemento, se impulsa además el establecimiento de una red de asesores y asesoras para promover la educación intercultural en los centros desde un planteamiento global y se crea la figura del mediador cultural en las oficinas de acogida para asesorar y orientar a los diferentes agentes de la comunidad educativa y colaborar en la búsqueda de consensos en las normas que han de regir la convivencia pacífica de los centros. Finalmente, se desarrollan iniciativas en el ámbito de los recursos pedagógicos elaborando, por ejemplo, guías para el trabajo de la educación intercultural, materiales para el aprendizaje del castellano y valenciano o creando premios para materiales dirigidos a la atención educativa a estudiantes inmigrantes.

\section{Política e integración del alumnado inmigrante en Cataluña}

Desde que a inicios de 1981 fueran transferidas a la Generalitat las competencias de educación se inició el camino de la estructura organizativa actual. La adaptación del alumnado extranjero ha experimentado diferentes fases desde la década de los noventa (Vila, 2011). En una primera fase, se crearon los denominados Talleres de Adaptación Escolar (TAE), agrupamientos formados exclusivamente por estudiantes extranjeros con la finalidad de llevar a cabo un aprendizaje acelerado del catalán como paso previo a la adaptación.

Aunque años atrás se había desarrollado el "Programa de Educación Compensatoria", en el año 2003 se elabora el Plan de Actuación para el Alumnado de 
Nacionalidad Extranjera 2003-2006 (PAANE). Este Plan contempla principalmente los aspectos relacionados con el aprendizaje de la lengua sin tener en cuenta otros fundamentales para la acogida como los emocionales, relacionales y de cohesión social.

El análisis de estas debilidades lleva en el año 2004 a la aprobación del "Plan para la Lengua y la Cohesión Social", enmarcado dentro del "Plan Interdepartamental de Inmigración". En este mismo año se crea la Subdirecció General de Llengua i Cohesió Social, con la voluntad de garantizar la igualdad de oportunidades para acceder a una educación de calidad, respetar la diversidad cultural y consolidar la lengua catalana como eje de un proyecto educativo plurilingüe, fundamentado en los valores de la convivencia, la equidad y la inclusión social y escolar de todo el alumnado. Además, a principios de 2008 se realiza una propuesta polémica: la creación de un sistema de acogida inmediata en espacios transitorios para el alumnado que llegaba una vez iniciado el curso escolar (Carrasco, Pàmies y Narciso, 2012; Simó, Pàmies, ColletSabé y Tort, 2014). La temporalidad de la estancia es la cuestión más importante pero la menos concretada y fue objeto de críticas diversas por el riesgo de segregación que encerraba.

Aparecen así en esta época las Aulas de Acogida, entendidas como un sistema mixto en el que el alumnado reparte su horario escolar entre el grupo-clase y dicha aula de acogida ubicada en el mismo centro. En la voluntad de integrar en torno a sus objetivos el trabajo de las instituciones escolares el Plan LIC (Lengua, Interculturalidad y Cohesión Social) apostaba por otros ejes estratégicos además de las ya mencionadas Aulas de acogida: el centro acogedor; el plan educativo de entorno, el equipo de asesoramiento en lengua, interculturalidad y cohesión social y la formación del profesorado (Besalú 2006; Garreta, 2011; Llevot, 2006).

En el año 2013 y de acuerdo a los principios de La Ley de Educación de Cataluña de 10 de julio de 2009 se desarrolla el "Plan para la reducción del fracaso escolar" que plantea realizar una ofensiva a favor del éxito escolar en seis años (2012-2018) con tres grandes objetivos (Departament d'Ensenyament, 2013): mejorar el nivel competencial de los alumnos, incrementar el rendimiento académico en la ESO y reducir la tasa de abandono escolar prematuro. Este plan presenta diversos ejes de actuación dentro de los cuales muchas medidas se enfocan a la atención a la diversidad sociocultural. Destacan en relación al tema que nos ocupa, la formación y orientación a los centros y al personal docente en aspectos de interculturalidad y de cohesión social por parte de los equipos de refuerzo y de asesoramiento en lengua, interculturalidad y cohesión social (ELIC), la realización de cursos telemáticos sobre la acogida del alumnado recién llegado o la debida dotación de aulas de acogida tanto en la Educación Primaria como en la Secundaria.

Parece interesante destacar, además, la implementación de Proyectos de convivencia trabajados en el aula, centro o entorno en los que se abordan cuestiones asociadas a los valores, actitudes, gestión de conflictos u organización de centro. 


\section{Política de integración del alumnado inmigrante en la Comunidad Autónoma de Andalucía}

Desde la Consejería de Educación de la Junta de Andalucía se canalizan actuaciones específicas, en coordinación con otras Consejerías (Salud y Bienestar, por ejemplo), para el fomento de actividades formativas relacionadas con la integración.

En los últimos años, cuatro han sido las actuaciones o planes de intervención que se han puesto en funcionamiento para dar respuesta a las necesidades de la población inmigrante: El "I Plan Integral para la Inmigración en Andalucía (2001-2004)", "El Plan Andaluz para la Inclusión Social (2003-2006)", el "II Plan Integral para la Inmigración en Andalucía (2006-2009) y el "III Plan Integral para la Inmigración en Andalucía Horizonte 2016", que ha sido aprobado mediante el Decreto 124/2014. El gobierno andaluz ha apostado en su política migratoria por los principios de universalidad e integración. En el Plan se presentan las distintas áreas de intervención, sus objetivos, alcance y los organismos encargados de su gestión. De entre las que se despliegan, son especialmente sensibles con el colectivo de menores inmigrantes, el área socio-educativa y el área socio-sanitaria. Dentro del área socioeducativa conviene resaltar que se prevén medidas en tres ejes: acogida e integración, enseñanza del español y mantenimiento de la cultura de origen. Además de los Programas de Acompañamiento Escolar y Refuerzo Educativo y la realización de convenios de colaboración con entidades locales, es preciso destacar que el III Plan contempla la continuidad de las Aulas Temporales de Adaptación Lingüística (ATAL) que ya se encontraban reguladas desde la Orden de 15 de enero de 2007. En efecto, se trata de programas de enseñanza y aprendizaje del español como lengua vehicular, vinculados a profesorado específico, que permiten la integración del estudiantado inmigrante en el centro y su incorporación a los ritmos y actividades de aprendizaje propios del nivel en el que se encuentren escolarizados según su edad y competencia curricular (Pegalajar, 2011).

En cuanto al proceso de escolarización, conviene resaltar que se busca facilitar la escolarización en cualquier época del año, de todo el alumnado perteneciente a familias inmigrantes. Para ello se han venido poniendo en marcha medidas como información a las familias y colectivos de inmigrantes sobre el proceso de escolarización y organización del sistema educativo en Andalucía, acceso a los servicios complementarios de comedores, transporte escolar, así como residencias escolares que permitan la continuación de los estudios postobligatorios. También se ha tratado de asegurar el proceso de escolarización mediante la dotación de recursos humanos y materiales extraordinarios en los centros que escolaricen un número significativo de alumnado inmigrante y que requieran medidas de compensación educativa. Aunque en menor medida también se han articulado otras estrategias, como disminución de la ratio y organizaciones de grupos de refuerzo y de apoyo educativo; formalización de convenios con entidades sin ánimo de lucro para establecer la figura del mediador cultural; incremento de la oferta de Programas de iniciación profesional tanto en Institutos de Educación Secundaria como a través de Convenios con Ayuntamientos y Asociaciones, etc. 
Finalmente, conviene destacar que el Plan contempla para cada uno de los objetivos específicos en cada una de las áreas, indicadores de evaluación que pretenden facilitar la revisión del mismo en los documentos técnicos de seguimiento y en las revisiones periódicas que se tiene previsto realizar.

\section{Discusión}

Nos encontramos ante tres Comunidades Autónomas que, en el seno del Estado español de las Autonomías, presentan particularidades, fruto de trayectos históricos, coyunturas económicas y políticas, intenciones educativas y sociales propias. Medidas y planes variados, con concreciones reales o apenas dibujadas, influidos por contextos en los que algunos factores han detentado un peso específico importante: la presencia, la procedencia, las implicaciones, las influencias y también la continuidad.

A pesar de las décadas de experiencia, la tradición de tratamiento específico a través de planes concretos de inmigración no ha dejado paso en la realidad estudiada, a una integración más "natural" en la propia estructura de actuación de las diferentes políticas. Es común una implicación interdepartamental, con responsabilidad y actuaciones de diferentes Consejerías, en las cuestiones relativas a la inmigración, que acaban concretándose en los mencionados planes estratégicos específicos que aúnan medidas de diferente corte y sesgo como hemos tenido ocasión de analizar en el estudio de casos. De este modo, en la Comunidad Valenciana son las Consejerías de Bienestar Social y la de Educación, Cultura y Ciencia las que generan los diferentes Planes autonómicos y locales y el marco de actuación viene de la mano del Plan Valenciano de Inmigración. En Cataluña esta realidad se acompañará del señalado Plan Interdepartamental de Inmigración. Para el caso andaluz volvemos a encontrarnos con la implicación de la Consejería de Salud y Bienestar Social, a través del área de servicios sociales, como promotoras de grandes Planes Integrales.

El protagonismo de los diferentes Departamentos o Consejerías educativas en la atención a la población escolar inmigrante se concreta para Valencia en la Conselleria d'Educació, Cultura i Ciència, mientras que en el caso catalán lo hace en el Departament d'Ensenyament. Una vinculación en los primeros años de la década pasada con los programas de compensación educativa podemos encontrarla también en los casos valenciano y catalán. Para Valencia, el vínculo entre atención a la población escolar inmigrante y educación compensatoria lo obtenemos en el marco de la Orden de 4 de julio de 2001 de la Conselleria d'Educació, Cultura i Ciència, que regula la atención al alumnado con necesidades educativas especiales.

El contexto lingüístico catalán tiene sin duda un claro influjo en la particularidad de las diferentes subdirecciones reconocidas como responsables en los procesos de atención a la realidad intercultural de las aulas y dependientes de la Consejería educativa, en concreto la Subdirecció General de Llengua i Cohesió Social. Las reestructuraciones posteriores encierran también una particular vivencia del peso lingüístico en la cohesión de la sociedad catalana y una adaptación a las nuevas demandas en el seno de los sistemas educativos europeos, cuando en 2011, con el marco legal de la Llei d'Educació de Catalunya de 10 de julio de 2009, se reestructura 
dicho Departament d'Enseyament. En esta línea, otra norma, fruto innegable de las tendencias evaluadoras internacionales, marcará las actuaciones, nos referimos al Pla per a la reducció del fracàs escolar (Departament d'Ensenyament 2013).

En las medidas puestas en marcha encontramos también puentes de acercamiento, coincidencias y diferencias notables. Entre los elementos comunes a la dinámica más propiamente educativa figuran la elaboración de planes de acogida e integración del alumnado; el desarrollo y promoción de la educación intercultural descrito para cada uno de los casos; la creación de aulas de acogida y talleres de adaptación escolar en Cataluña, concretadas en las aulas PASE del modelo valenciano o el apoyo al aprendizaje de la lengua a través, desde 2007, de las Aulas Temporales de Adaptación Lingüística en el contexto andaluz; elemento común encontramos en los tres modelos estudiados también en la formación ofertada a los profesionales de los centros más afectados numéricamente por la población inmigrante, así como a la existencia de asesoramiento especializado en relación a las prácticas interculturales. La catalogación numérica de la red de centros marca la realidad de las medidas puestas en marcha en el ámbito de las comunidades, algo que queda especialmente explícito para el caso de la Comunidad Valenciana. También se encuentra presente en las tres realidades estudiadas, la elaboración y publicación de guías de recursos didácticos y materiales y su difusión a través de la red. A todo ello se unen los diferentes programas de refuerzo con medidas como las de acompañamiento en primaria y secundaria comunes a los tres contextos, en muchos casos centrados en los cambios de ciclo y en la iniciación profesional como ocurre en Andalucía.

Los vínculos con el entorno, entendido en un sentido más amplio, incluyen la potenciación de Planes Educativos de Entorno que relatábamos para el caso catalán, que encuentra correspondencia en medidas valencianas concretadas en las Oficinas de acogida y atención al alumnado inmigrante y su familia (desde 2001) con mediadores culturales que desempeñan precisamente esas funciones de relaciones con la comunidad o también los mediadores culturales andaluces. La intervención con las familias forma parte igualmente de la oferta común de medidas en los tres modelos.

La riqueza del modelo valenciano y su particularidad viene dada básicamente por la insistencia en la vinculación de sus propuestas a los objetivos de estudio, con la creación de su propio observatorio de la inmigración, la investigación y difusión de las prácticas exitosas materializadas en la convocatoria de proyectos de investigación e innovación educativa y premios de la Comunitat dirigidos a materiales destinados a la atención educativa al alumnado inmigrante. Elaborado y transversalmente trabajado se encuentra el proyecto lingüístico en Cataluña, donde también hallamos una incidencia mayor de las temáticas e iniciativas innovadoras en el ámbito pedagógico. La realidad catalana da también muestras de los esfuerzos por aunar y vincular las actuales inquietudes de evaluación educativa con medidas concretas contra la deserción o el abandono escolar. En Andalucía localizamos algunos rasgos distintivos de un modelo que pretende la articulación con las realidades vitales de la población inmigrante más allá del estricto velar por los niveles educativos obligatorios, con previsiones relativas al acceso a servicios complementarios como residencias escolares para poder continuar estudios después de la enseñanza obligatoria. Resultan también 
especialmente atractivos en este contexto los programas que compaginan el refuerzo en la adquisición de la lengua vehicular de la enseñanza con la promoción de la lengua materna.

\section{Conclusiones comparadas. Limitaciones del estudio y, en su caso, prospectiva}

La crisis económica ha supuesto, entre otros muchos aspectos, el incremento notable de diversas acciones a nivel local y una mayor coordinación en el diseño de políticas y actuaciones desde cada gobierno autonómico hacia los colectivos en riesgo de marginación social y cultural. Esta realidad, afectando al tema que nos ocupa, no forma parte de las políticas educativas concretas de atención al alumnado inmigrante, pero puede convertirse en motor de una tendencia que implique una mayor coordinación en la atención integral de las necesidades del alumnado.

La discusión reflejada en el apartado anterior permite dar cuenta de algunos marcos generales que exceden el propiamente educativo y hacer un recorrido de líneas prioritarias de actuación, semejanzas y diferencias entre medidas adoptadas en unos y otros contextos. Recorrido que, para las políticas más concretamente educativas, encuentra en el marco legislativo de la ya referida Ley Orgánica para la mejora de la calidad educativa (LOMCE), un punto de partida que asocia de forma expresa en su preámbulo los términos equidad y calidad, dando prioridad, según se expresa textualmente, a "la eliminación de cualquier atisbo de desigualdad". Sin embargo, este término de la equidad en el tratamiento de las políticas educativas de atención al alumnado inmigrante parece asociarse conforme avanza el texto a las cuestiones de equidad social y su vínculo con la competitividad del país, es decir, como producto del sistema, más que como condición para su buen funcionamiento. En cualquier caso, será el tiempo el que nos permita vislumbrar si la efectiva implantación de la LOMCE potencia la materialización, en aras de la calidad, de un modelo educativo inclusivo o, por el contrario, selectivo y segregador.

El trabajo realizado encuentra, de forma más concreta en la literatura existente, contraste con los logros y resultados de cada una de estas prácticas de atención al alumnado inmigrante. Buen ejemplo de los análisis que posibilita esta brecha pudieran ser estudios como los de Essomba (2014), García, Rubio y Bouachra (2015) o, circunscritos a contextos más concretos, la investigación de Fernández (2013) para el caso andaluz o la de Simó, Pàmies, Collet-Sabé y Tort (2014), para el caso catalán.

En el análisis de la realidad es posible encontrar coincidencias en cuanto a los retos que pueden marcar en cierto modo algunas sugerencias para las agendas de trabajo y con las que nos gustaría concluir. Por un lado, la literatura referida se hace eco, como también lo hace la LOMCE, de la pluralidad de agentes implicados en la transformación educativa. Mientras que la ley educativa insiste en el papel de empresas, asociaciones, sindicatos, organizaciones no gubernamentales, familias y otras formas de manifestación de la sociedad civil, desde el ámbito académico se reclama el peso específico de familia y factores extraescolares (Fernández, 2013) o desde los planteamientos de Essomba (2014) se solicita, con una mayor profundidad y 
amplitud de miras, la necesidad de paso de las políticas educativas basadas en la escuela a las políticas educativas basadas en la comunidad y de las políticas educativas de carácter sectorial a las políticas educativas de carácter sistémico. No es casualidad que en este sentido la experiencia catalana descrita sea recogida como buena práctica.

Por otro lado y vinculada con lo anterior, nos gustaría destacar otra tendencia que nos parece relevante, asociada la explicación concreta de estas prácticas en los contextos estudiados; en concreto, las referencias a las perspectivas relacionales que insisten en el peso específico de la búsqueda de vínculos como clave para el éxito a la hora de proporcionar una calidad adecuada en el proceso de escolarización de este alumnado: "la innovación pasa porque quienes diseñan e implementan políticas superen una perspectiva estructural-funcional del sistema educativo, y se imbuyan en una perspectiva más relacional, en la cual la clave del éxito no reside en los componentes del sistema sino en el sistema de relaciones que estos establecen entre sí, dentro y fuera del contexto escolar" (Essomba, 2014: 22). En este marco se encuentra ese concepto de comunidad que permite el intercambio de experiencias entre el profesorado, como auténticas comunidades de aprendizaje, y también entre el alumnado mediante la activación de estrategias y mecanismos que permitan aprender unos de otros, compartir las propias experiencias con un papel protagonista que los sitúe como artífices del cambio. También en esta línea han quedado descritas las prácticas variadas según contextos.

Finalmente, sí resulta significativo, por las implicaciones con nuestro objeto de estudio, el hecho de que uno de los debates más polémicos que se ha producido entre Consejeros de Educación autonómicos y el Ministro en el trabajo de preparación y discusión de la LOMCE ha sido el del respeto a las lenguas cooficiales, especialmente en el caso catalán. Entre los factores que intervienen en la mejor adquisición de la lengua vehicular del centro hay investigaciones que destacan la importancia de prestar atención a la lengua que aporta el propio alumnado. Por ejemplo, Vila (2004) indica que es básico desarrollar los medios necesarios para que la inmigración mantenga su propia lengua, entendiendo que un buen dominio de la lengua propia y su valorización conllevan una mejor disposición y habilidades para el aprendizaje de una nueva lengua. Por ello parece necesario incluir y/o potenciar programas de aprendizaje de las lenguas maternas con mayor demanda en los centros, con la finalidad de facilitar al alumnado su conocimiento reglado y fomentar actitudes positivas hacia la diversidad cultural y lingüística.

Atender a la diferencia, eje supuesto de todas y cada una de las actuaciones, pasa, en nuestros sistemas, por una exigencia común: dotar de la lengua vehicular. El mismo proceso de unificación lingüística que en la constitución de nuestros sistemas educativos nacionales se convirtiera no tanto en garante de la diferenciación, sino más bien en elemento generador del sentido de pertenencia a los decimonónicos y entonces nacientes estados-nación liberales (Ayala y González, 1998). Hay mucho sentido en el matrimonio que la Generalitat apunta en sus Consejerías: lengua y cohesión social. Efectivamente, se trata de "cohesión", a través de la lengua común; de ahí su importancia y los desvelos por ella. Podemos pues, en algún momento, dejar de 
enarbolar la bandera del respeto a la diferencia y asumir el objetivo último que tiene en la pretendida cohesión social más andamios y logros.

Es de esperar que las buenas prácticas que como tal se han mostrado, reconocido y materializado gocen del respaldo prioritario que un asunto de tal envergadura merece, con la incorporación paulatina del peso específico de relaciones y redes sociales que contribuyan a una tarea que debiera conmovernos y comprometernos como educadores y ciudadanos.

\section{Referencias bibliográficas}

AJA, E.; ARANGO, J.; OLIVER, J. (Eds.) (2012). La hora de la integración. Barcelona: Bellaterra.

ALCALDE, R. (2008). Los programas de actuación educativa orientados al alumnado de origen extranjero: ¿modelos de atención a la diversidad cultural o a la igualdad educativa? Revista de Educación, 345, 207-228.

ALEGRE, M.A.; BENITO; R. Y GONZÁLEZ, S. (2012). Experiencias escolares, iniciales del alumnado inmigrado: comienzos que marcan. Educación XXI, 15 (2), 137-158. DOI: http://dx.doi.org/10.5944/educxx1.15.2.130.

ARROYO, M.J. (2012). Las aulas de inmersión lingüística para alumnado inmigrante en el marco de la escuela inclusiva: Algunas propuestas de mejora. Tendencias Pedagógicas, 19, 25-42.

AYALA, A. Y GONZÁLEZ HERNÁNDEZ, A. (1998). Estado y educación en la teoría de la ciudad -Estado: la actualidad de dos antecedentes histórico-culturales. Anales de Pedagogía, 16, 9- 20.

BESALÚ, X. (2006). El Plan para la Lengua y la Cohesión Social (Plan LIC) del gobierno de Cataluña. Revista Interuniversitaria de Formación del Profesorado, 58 (1), 45-68.

BRAY, M. \& THOMAS, R. (1995). Levels of Comparison in Educational Studies: Different Insights from Different Literatures and the Value of Multilevel Analysis. Harvard Educational Review, 65 (3), 472-490.

CARBONELL, R. (2006). La respuesta educativa en la Comunitat Valenciana. En J. M. Senent y P. Villar (Eds.). Miradas y encuentros entre las orillas del Mediterráneo. Actas de las Jornadas Mediterráneas sobre inmigración (pp. 173184). Valencia: Universitat de València.

CARRASCO, S.; PÀMIES, J. Y NARCISO, L. (2012). A propósito de la acogida de alumnado extranjero. Paradojas de la educación inclusiva en Cataluña (España). Revista Latinoamericana de Educación Inclusiva, 6 (1), 105-122.

COMISIÓN DE LAS COMUNIDADES EUROPEAS (2008). Libro Verde. Inmigración y movilidad: retos y oportunidades de los sistemas educativos de la 
UE. $\operatorname{COM}(2008) \quad 423$ final. Recuperado de: http://eurlex.europa.eu/LexUriServ/LexUriServ.do?uri=COM:2008:0423:FIN:ES:PDF.

CROSSLEY, M. (2008). Bridging cultures and traditions for educational and international development: comparative research, dialogue and difference. International Review of Education, 54, 319-336.

DECRETO 124/2014, de 2 de septiembre por el que se aprueba el III Plan Integral para la Inmigración en Andalucía Horizonte 2016. Boletín Oficial de la Junta de Andalucia, núm. 172, de 4 de septiembre 2014, pp. 5 a 6.

DEL OLMO, M. (2012). Buenas prácticas, ¿desde el punto de vista de quién? Una contribución a la controversia sobre las aulas de enlace. Revista de Educación, 358, 111-128. DOI: 10-4438/1988-592X-RE-2012-358-185

DEPARTAMENT D'ENSENYAMENT (2013). Ofensiva del país a favor de l'èxit escolar. Pla per a la reducció del fracàs escolar a Catalunya 2012-2018. Barcelona: Generalitat de Catalunya.

ESSOMBA, M.A. (2014). Políticas de escolarización del alumnado de origen extranjero en el estado español hoy. Análisis y propuestas. Revista Electrónica Interuniversitaria de Formación del Profesorado, 17 (2), 13-27. DOI: http://dx.doi.org/10.6018/reifop.17.2.198771

ETXEBERRIA, F. Y ELOSEGUI, K. (2010). Integración del alumnado inmigrante: obstáculos y propuestas. Revista Española de Educación Comparada, 16, 235-263.

EURYDICE (2009). La integración del alumnado inmigrante en Europa. Bruselas: Eurydice. Recuperado de: http://eacea.ec.europa.eu/education/eurydice/documents/thematic_reports/101ES.p df.

FERNÁNDEZ F. (2013). Desigualdad educativa. El alumnado inmigrante en el sistema educativo andaluz: un estudio de caso. Praxis Sociológica, 17, 185-204.

GARCÍA, F.J. y CARRASCO, S. (Eds.) (2011). Población inmigrante y escuela: conocimientos y saberes de investigación. Madrid: Secretaría General Técnica del Ministerio de Educación.

GARCÍA, F.J., GRANADOS, A., OLMOS, A. Y MARTÍNEZ, R. (2014). Cuando no hablamos de integración: análisis de la imagen del alumnado "inmigrante" en la prensa en España. Estudios sobre el mensaje periodístico, 20 (2), 1081-1101. DOI: http://dx.doi.org/10.5209/rev_ESMP.2014.v20.n2.47051

GARCÍA, F.J. Y RUBIO, M. (2013). «Juntos pero no revueltos»: Procesos de concentración escolar del «alumnado extranjero» en determinados centros. Revista de Dialectología y Tradiciones Populares, 68 (1), 7-31.

GARCÍA, F.J., RUBIO, M. Y BOUACHRA, O. (2008). Población inmigrante y escuela en España: un balance de investigación. Revista de Educación, 345, 23-60. 
GARCÍA, F.J.; RUBIO, M. Y BOUACHRA, O. (2015). Immigrant students at school in Spain: constructing a subject of study. DVE Domovini. Two Homelands, 41, 3546.

GARRETA, J. (2011). La atención a la diversidad cultural en Cataluña: exclusión, segregación e interculturalidad. Revista de Educación, 355, 213-233. DOI: 104438/1988-592X-RE-2011-355-022

GOENECHEA, C. (2005). Estudio empírico de la situación educativa del alumnado extranjero en Galicia. Revista de Investigación Educativa, 23 (1), 187-204.

GRAÑERAS, M.; Vázquez, E.; Parra, A.; Rodríguez, F.; Madrigal, A. y Vale, P. (2007). La atención lingüística al alumnado extranjero en el sistema educativo español: normativa, actuaciones y medidas. Revista de Educación, 343, 149-174.

HALLS, H.D. (1990). Comparative Education. Contemporary issues and trends. London: Jessica Kingsley.

HUDDLESTON, T. \& NIESSEN, J. (2011). Migrant Integration Policy Index.Bruselas: British Council y Migration Policy Group. DOI: http://www.mipex.eu/.

LARSEN, M. A. (2010). New Thinking in Comparative Education: Editorial introduction. En M. A. Larsen (Ed.). New Thinking in Comparative Education. Honouring Robert Cowen (pp. 1-15). Rotterdam: Sense Publish.

LÊ THÀNK KÔI (1981). L'éducation Comparée. Paris: Armand Collin.

LEY 12/2009, de 10 de julio, de Educación [2009/13038]. BOE núm. 189, de 6 de agosto de 2009, pp. 67041 a 67143.

LEY ORGÁNICA 8/2013, de 9 de diciembre, para la mejora de la calidad educativa. BOE No 295, de 10 de diciembre de 2013, pp. 97858 a 97921.

LLEVOT, N. (Ed.) (2006). La educación intercultural: discursos y prácticas. Lleida: Universitat de Lleida.

LLORENT, V. y TERRÓN, M.T. (2013). Políticas socioeducativas de integración de los inmigrantes en Alemania, España y Francia. Pedagogía Social. Revista Interuniversitaria, 21. Recuperado de: http://dx.doi.org/10.7179/PSRI_2013.21.5.

MANZÓN, M. (2007). Comparing Places. En M. Bray, B. Adamson \& M. Mason (Eds.). Comparative Education Research. Approaches and Methods (pp. 85-122). Hong Kong: Comparative Education Research Centre and Springer.

MARTÍNEZ DE LIZARRONDO, A. (2009). Políticas autonómicas de integración de inmigrantes: la educación. Revista Española de Educación Comparada, 15, 251276.

MARTÍNEZ USARRALDE, M.J. (2008). Una mirada comparada al contexto internacional: revisión de la realidad del mediador cultural en algunos países. El caso de Canadá, Italia, Suecia y Portugal. Revista de Educación, 345, 353 - 376. 
MARTÍNEZ USARRALDE, M.J. (Ed.) (2010). Y para muestra ... Políticas educativas de inmigración y modelos de escuela que practican la interculturalidad. Valencia: Universitat de València.

MINISTERIO DE EDUCACIÓN Y CIENCIA (2014). Datos y cifras del curso escolar 2014/2015. Madrid: MEC.

NOAH, H.J. (1990). Usos y abusos de Educación Comparada. En P.G. Albatch y G.P. Kelly (1990). Nuevos enfoques en Educación Comparada (pp. 177-190). Madrid: Biblioteca Mayor Mondadori.

OECD (2012). Untapped skills. Realising the potential of immigrant students. Paris: OECD.

ORDEN DE 4 DE JULIO DE 2001, de la Conselleria de Cultura i Educació, por la que se regula la atención al alumnado con necesidades de compensación educativa. [2001/X6756]. DOCV núm. 4404, de 17 de julio de 2001, pp. 16.282 a 16.293.

ORDEN DE 15 DE ENERO DE 2007 por la que se regulan las medidas y actuaciones a desarrollar para la atención del alumnado inmigrante y, especialmente, las Aulas Temporales de Adaptación Lingüística. BOJA núm. 33, de 14 de febrero de 2007, pp. 7 a 11.

PALAUDÀRIAS, J. Y GARRETA, J. (2008). La acogida del alumnado de origen inmigrante: un análisis comparado desde la situación en Cataluña. Revista Española de Educación Comparada,14, 49-78.

PEGALAJAR, M. C. (2011). Importancia de las aulas temporales de adaptación lingüística para la enseñanza del español en alumnado inmigrante en el sistema educativo andaluz. Actas del I Congreso Internacional sobre Migraciones en Andalucia (pp. 513-516). Sevilla: Universidad de Sevilla.

PHILLIPS, D. (2000). Learning from Everywhere in Education, some perennial problems revisited with reference to British interest in Germany. Comparative Education, 36, 297-307.

SADLER, M. (1900). How far can we learn anything of practical value from the study of foreign systems of education? Guildford: Survey Office.

SCHRIEWER, J. (1990). The method of Comparison and the need for Externalization, Methodological Criteria and Sociological Concepts. En J. Schriewer \& B. Holmes (Eds.). Theories and Methods in Comparative Education (pp. 25-83). Frankfurt and Main: Peter Lang.

SCHRIEWER, J. (2010). Comparación y explicación entre causalidad y complejidad. En J. Schriewer y H. Kaelble (Comps.). La comparación en las ciencias sociales e históricas (pp. 17-62). Barcelona: Octaedro.

SIMÓ, N.; PÀMIES, J.; COLLET-SABÉ, J. Y TORT, A. (2014). La acogida educativa en los centros escolares en Cataluña: más allá de los recursos específicos para el alumnado de nueva incorporación. Revista Complutense de Educación, 25 (1), 177-194. DOI: http://dx.doi.org/10.5209/rev_RCED.2014.v25.n1.41410. 
SIQUES, C., VILA, I. Y PERERA, S. (2009). Percepciones y actitudes escolares del alumnado extranjero y del profesorado: un estudio empírico en las aulas de acogida de Catalunya. Electronic Journal of Research in Educational Psychology, 7 (17), 103-132.

VEGA, A.M. (2005). Integración e interculturalidad en el medio escolar riojano. Un análisis exploratorio. Estudios sobre Educación, 8, 61-116.

VILA, I. (2004). Diversidad, escuela e inmigración. Aula de innovación educativa, $131,62-66$.

VILA, I. (2011). Balanç de la política lingüística a l'educació escolar dels governs de Catalunya (2004-2011). En J.M. Masjuan (Coord.). Sociedad Catalana (pp. 295323). Barcelona: Institut d'Estudis Catalans y Associació Catalana de Sociologia.

\section{Correspondencia con los autores}

María Jesús MARTÍNEZ-USARRALDE

Departament d' Educació Comparada i Història de 1' Educació.

Facultat de Filosofia i Ciències de 1' Educació.

Avgda. Blasco Ibáñez, 30.

46020 València.

e-mail: m.jesus.martinez@uv.es

Carmen María FERNÁNDEZ-GARCÍA

Facultad de Formación de Profesorado y Educación

C/ Aniceto Sela s/n. Despacho 235.

33005 Oviedo (Asturias).

e-mail: fernandezcarmen@uniovi.es

Amalia AYALA-DE LA PEÑA

Facultad de Educación.

Dpto. de Teoría e Historia de la Educación

Campus de Espinardo s/n.

30100 Espinardo (Murcia)

e-mail: amayala@um.es 